\title{
Some Observations on the Envelope of an Influenza Virus
}

\author{
By JUNE D. ALMEIDA AND A. P. WATERSON \\ Department of Medical Microbiology, St Thomas's Hospital Medical School, \\ London, S.E.1
}

(Accepted for publication 16 August 1966)

\begin{abstract}
SUMMARY
Micrographs of the envelope of fowl-plague virus revealed an arrangement of subunits each one of which was surrounded by either five or six others. The similarity of this arrangement to that of a cubic virus capsid has led us to consider which components of morphologically different viruses fulfil similar functions.
\end{abstract}

\section{INTRODUCTION}

A virus particle consists of two parts, the essential nucleic acid and a means of protecting this from the environment. It was predicted that the protective covering would be built up of identical repeating subunits (Crick \& Watson, 1956). Early $\mathrm{X}$-ray diffraction studies suggested that these units would be arranged symmetrically (Hodgkin, 1949) and that the symmetry would be one of two types, helical or cubic. The application of negative-staining to virus particles shortly after this revealed pictorially both types of symmetry (Horne \& Wildy, 1961). Viruses which had by previous techniques been considered to be spherical were now seen to be icosahedral, i.e. belonged to the cubic group, and particles which had been described as rod-shaped were now seen to have helically-arranged subunits. The cubic arrangement was found for DNA and for RNA viruses and can be found among bacterial, plant and animal viruses. The simple helical arrangement, on the other hand, seems to be confined mainly to RNA viruses, and, of even greater importance in the present connexion, has not been found in the simple form among animal viruses. Helical symmetry, however, is frequently present among animal viruses, the simple RNA and protein arrangement (ribonucleoprotein) being found enclosed within an additional lipoprotein envelope. The overall form of this outer envelope is usually irregular, and viruses such as influenza are usually described as being pleomorphic. The lipoprotein covering of such viruses seems to contain both viral and host material (Cruickshank, 1964). Until now it has not been looked on as conforming to the requirements of virus structure - that is, made up of identical repeating subunits identically arranged in a helical virus, or with each subunit surrounded by either six or five others in a cubic virus (Caspar \& Klug, 1962).

\section{RESULTS}

While working with fowl-plague virus, an influenza A virus (Almeida, Himmelweit \& Isaacs, 1966), we obtained micrographs which suggested that, although not conforming to the arrangement found in either helical or cubic viruses, the envelope of pleomorphic viruses such as the myxoviruses may bear a much closer resemblance to these arrangements than was previously suspected. 
Plate 1, fig. 1., shows a negatively-stained fowl-plague virus particle with a completely regular array of subunits on the surface. Particles showing such complete regularity of arrangement were found only with difficulty and probably resulted from a fortunate combination of staining conditions and particle orientation. On the other hand, particles showing regular subunit arrangement over small areas could be found frequently and confirmed that the phenomenon was a real one and not an artifact formed by local conditions (Pl. 1, fig. 2). Like the cubic capsid (Pl. 1, fig. 3) the fowl-plague envelope illustrated in Pl. 1, fig. 1, is built up of subunits surrounded by either five or six other subunits. The difference between them lies in the fact that while the distribution of 'fives' and 'sixes' is fixed for the adenovirus capsid, it is random for the fowl-plague envelope. (Pl. 2, fig. 5)

This finding, that the envelope of a myxovirus resembles more closely than had been supposed the capsid of a cubic virus, led us to consider which components of morphologically distinct viruses-for example, measles and poliovirus-correspond most closely with regard to structure and function. Of course, there is no doubt that the helical ribonucleoprotein which constitutes the virion (the entire infective virus) in plant viruses, such as tobacco mosaic virus, bears a marked similarity to the internal component of a virus such as measles. They both consist of RNA and protein: in both the protein is arranged helically and the diameter of both is the same. The morphology of the ribonucleoprotein of measles, though usually seen as a herringbone pattern indicative of an extended helix, can nevertheless sometimes be seen in a much more straight and compressed form, very similar to the tobacco mosaic virus rod (PI. 1, fig. 4).

However, when the two are compared biologically, the roles of the two proteins are markedly different The tobacco mosaic virus protein is in direct contact with the environment and is apparently capable of protecting the nucleic acid from it, whereas the measles helix is separated from the environment by the envelope, and it seems improbable that the ribonucleoprotein should be capable of survival in the environment as an infective entity when not surrounded by this envelope. Again, when compared in their capacity as antigens, the tobacco mosaic virus protein will cause the production of antibodies which neutralize the virus, but the internal myxovirus protein will not (Schäfer, 1957). This means that, when compared biologically, the simple cubic virus bears the same relationship to the helical protein of tobacco mosaic virus as it does to the outer envelope of animal viruses with an internal helical component.

It follows that that, in terms of biological function, if not of morphology alone, it is the coverings presented to the environment that correspond, and the flexible helical ribonucleoprotein of the myxoviruses, inside its envelope, corresponds with the contents of the simple cubic virus capsid. This leads to the question of whether cubic capsids contain an internal protein in association with the nucleic acid. The absence of such a protein is by no means certain, and its presence would of course heighten the resemblance. One virus with a regular capsid (vesicular stomatitis virus) has now been shown to contain within its capsid a ribonucleoprotein helix (Brown, Cartwright \& Almeida, 1966); also, an arbovirus (Venezuelan equine encephalomyelitis), which may have a symmetrical cubic capsid, has been shown to contain a helix (Klimenko et al. 1965).

A further corollary is that the myxovirus envelope and the cubic capsid have more in 
common with each other than has been realized hitherto (Pl. 1, figs. 1, 3). Admittedly the myxovirus envelope contains lipid and carbohydrate, which are lacking from the cubic capsid, and is asymmetrical. On the other hand, this envelope is the basic means of protecting the internal nucleic acid, it is in contact with the environment, and it, rather than protein of the ribonucleoprotein, is the immunogen. In all of these respects the myxovirus envelope parallels the function of the cubic virus capsid.

It appears therefore that animal viruses may be regarded as having two components: first, the nucleic acid, which in some cases, e.g. the myxoviruses and vesicular stomatitis virus, has helically-arranged protein associated with it; secondly, the capsid or envelope, which consists essentially of protein units arranged as hexamers or pentamers, and which surrounds and protects the contained nucleic acid or nucleoprotein. This protective apparatus of 'fives' and 'sixes' can be arranged in either a symmetrical or a pleomorphic manner and can be built solely of protein or may have additional lipid and carbohydrate. The term 'nucleo-capsid' (Caspar et al. 1962) used for both the virion of simple cubic viruses and the ribonucleoprotein of myxoviruses is therefore misleading, being applied to two functionally different entities. If this reasoning is valid, then the distinction into cubic and helical would not be as meaningful as a division into cubic and pleomorphic. This would then compare analogous structures that appear to be constructed in a similar manner.

We are indebted to Dr H. G. Pereira for supplying us with the fowl-plague virus.

\section{REFERENCES}

Almeida, J. D., Himmelweit, F. \& IsaAcs, A. (1966). Studies on the intracellular haemagglutinin component of fowl plague virus and other myxoviruses. J. gen. Microbiol. 45, 153.

Brown, F., Cartwright, B. \& Almeida, J. D. (1966). The antigens of vesicular stomatitis virus. I. Separation and immunogenicity of three complement fixing components. J. Immunol. 96, 537.

Caspar, D. L. D., Dulbecco, T., Klug, A., Lwoff, A., Stoker, M. G. P., Tournier, P. \& Wildy, P. (1962). Proposals. Cold Spring Harb. Symp. quant. Biol. 27, 49.

Caspar, D. L. D. \& Klug, A. (1962). Physical principles in the construction of regular viruses. Cold Spring Harb. Symp. quant. Biol. 27, 1.

Crick, F. H. C. \& WATSON, J. D. (1956). Structure of small viruses. Nature, Lond. 177, 473.

Cruickshank, J. G. (1964). The structure of myxoviruses and its biological significance. In Cellular Biology of Myxovirus Infections. Ed. by G. E. W. Wolstenholme and J. Knight, p. 5. London: Churchill.

Hodgkin, D. C. (1949). X-ray analysis and protein structure. Cold Spring Harb. Symp. quant. Biol. 14, 65.

HORNE, R. W. \& WILDY, P. (1961). Symmetry in virus architecture. Virology 15, 348.

Klimenko, S. M., Yershow, F. I., Gofman, Y. P., Nabantikov, A. P. \& Zhdanov, V. M. (1965). Architecture of Venezuelan equine encephalomyelitis virus. Virology 27, 125.

SCHÄFER, W. (1957). Units isolated after splitting fowl plague virus. In The Nature of Viruses. Ed. by G. E. W. Wolstenholme and E. C. P. Millar, p. 91. London: Churchill.

\section{EXPLANATION OF PLATES}

\section{Plate 1}

Electron micrographs of negatively-stained preparations, all at $\times 415,000$.

Fig. 1. An unusual micrograph of a fowl-plague virus particle, showing the arrangement of subunits on its surface. The majority of these subunits are surrounded by six others, but a small, randomly located number have only five others round them. The arrow points to a location where a subunit surrounded by five others can be clearly seen. The centre-to-centre spacing of the subunits, about $70 \AA$, in this particle is almost identical with that of the capsomeres of the adenovirus particle shown in fig. 3 . 
Fig. 2. Subunit arrangements of the kind shown here are frequently encountered in preparations of influenza-type viruses. Although not as regular as the subunit array in fig. 1, it is still possible to see a geometric arrangement on the surface.

Fig. 3. An adenovirus particle viewed along a two-fold axis. It is similar to fig. 1 inasmuch as the capsid is composed of subunits surrounded by either five or six others, but dissimilar in that the distribution of these is rigidly fixed.

Fig. 4. A length of the ribonucleoprotein component of measles virus. More usually, micrographs of this structure show a herringbone arrangement, but in this case the helix has remained compressed and shows a strong resemblance to helical plant viruses (e.g. tobacco mosaic virus).

\section{Plate 2}

Fig. 5. Using 5- and 6-sleeve connectors, model ( $a$ ) was built to fulfil icosahedral requirements, that is, a 5-sleeve connector at each apex and 6-sleeve connectors in between, to give a total of 92 subunits. Model $(b)$ is built of randomly distributed 5-and 6-sleeve connectors. Although the overall appearance of the two models is quite different, the basic arrangement of each is the same and differs only in the presence or absence of symmetry. 

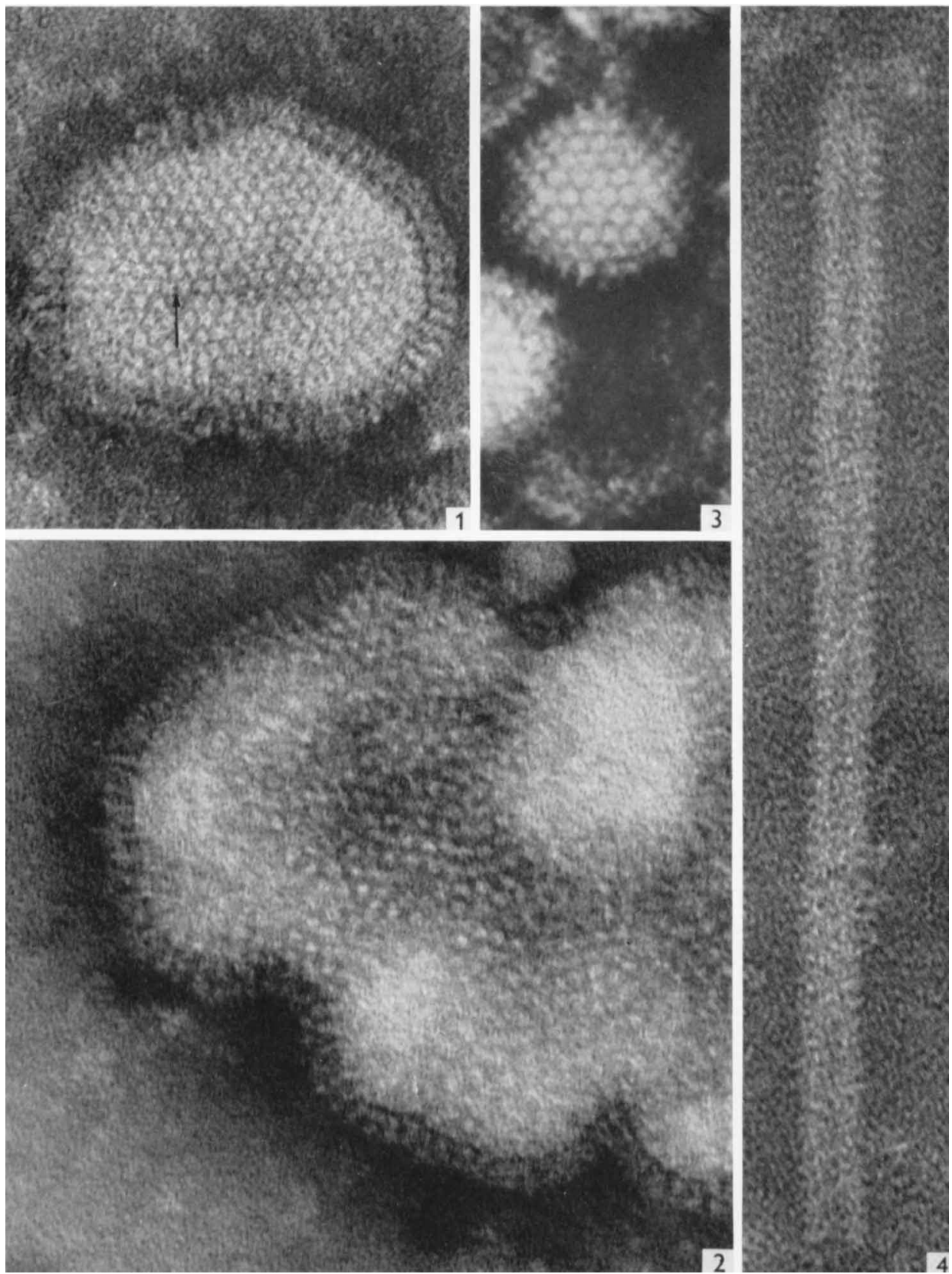

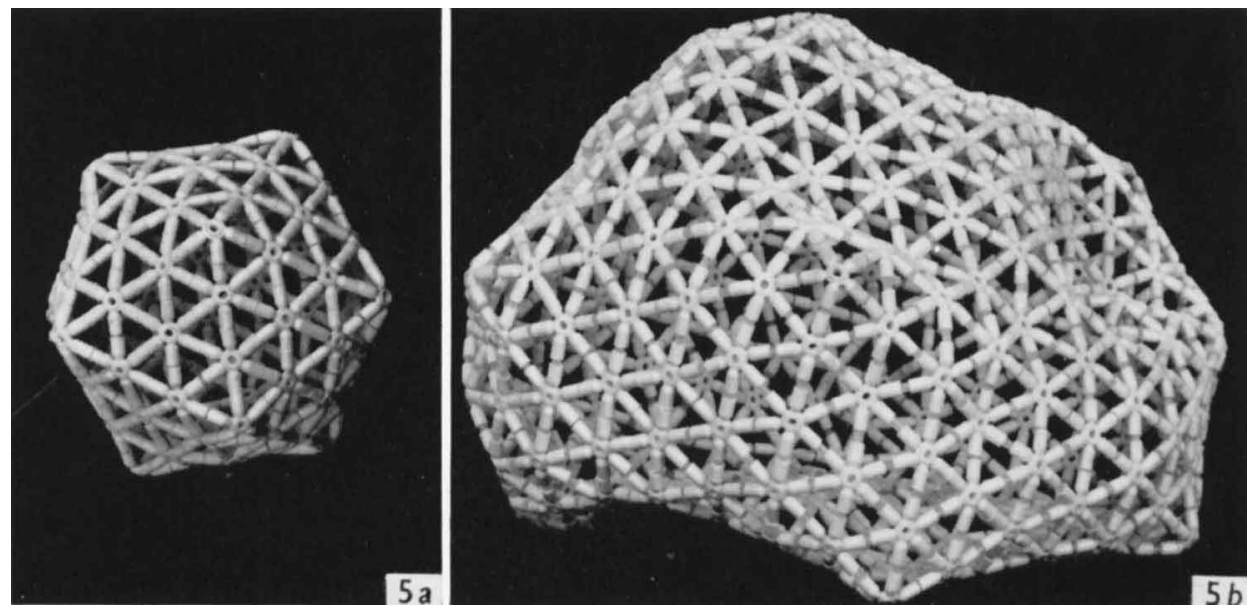